\title{
Droop-Free, Reliable, and High-Power InGaN/GaN Nanowire Light- Emitting Diodes for Monolithic Metal-Optoelectronics
} \author{
Chao Zhao, ${ }^{\dagger}$ Tien Khee Ng, ${ }^{*}{ }^{\dagger}$ Rami T. ElAfandy, ${ }^{\dagger}$ Aditya Prabaswara, ${ }^{\dagger}$ Giuseppe Bernardo Consiglio,
} Idris A. Ajia, ${ }^{\S}$ Iman S. Roqan, ${ }^{\S}$ Bilal Janjua, ${ }^{\dagger}$ Chao Shen, ${ }^{\dagger}$ Jessica Eid, ${ }^{\|}$Ahmed Y. Alyamani, ${ }^{\ddagger}$ Munir M. El-Desouki, ${ }^{\ddagger}$ and Boon S. Ooi* ${ }^{\dagger}$

${ }^{\dagger}$ Photonics Laboratory, King Abdullah University of Science and Technology (KAUST), Thuwal 23955-6900, Saudi Arabia

${ }^{\ddagger}$ National Center for Nanotechnology, King Abdulaziz City for Science and Technology (KACST), Riyadh 11442-6086, Saudi Arabia

${ }^{\S}$ Physical Sciences and Engineering Division, King Abdullah University of Science and Technology (KAUST), Thuwal 23955-6900, Saudi Arabia

"Solar and Photovoltaic Engineering Research Center, King Abdullah University of Science and Technology (KAUST), Thuwal 23955-6900, Saudi Arabia

\section{Supporting Information}
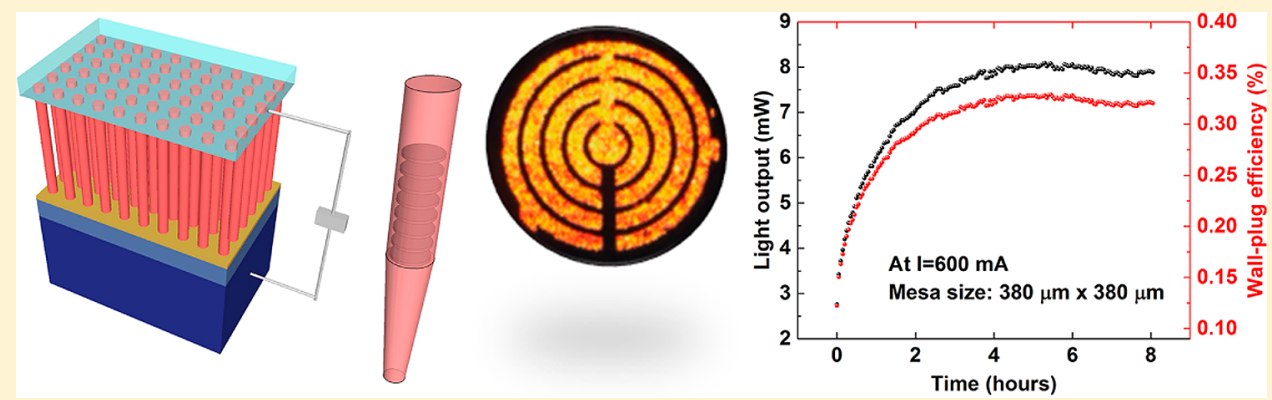

ABSTRACT: A droop-free nitride light-emitting diode (LED) with the capacity to operate beyond the "green gap" has been a subject of intense scientific and engineering interest. While several properties of nanowires on silicon make them promising for use in LED development, the high aspect ratio of individual nanowires and their laterally discontinuous features limit phonon transport and device performance. Here, we report on the monolithic integration of metal heat-sink and droop-free InGaN/GaN quantum-disks-in-nanowire LEDs emitting at $\sim 710 \mathrm{~nm}$. The reliable operation of our uncooled nanowire-LEDs (NW-LEDs) epitaxially grown on molybdenum was evident in the constant-current soft burn-in performed on a $380 \mu \mathrm{m} \times 380 \mu \mathrm{m} \mathrm{LED}$. The square LED sustained $600 \mathrm{~mA}$ electrical stress over an $8 \mathrm{~h}$ period, providing stable light output at maturity without catastrophic failure. The absence of carrier and phonon transport barriers in NW-LEDs was further inferred from current-dependent Raman measurements (up to $700 \mathrm{~mA}$ ), which revealed the low self-heating. The radiative recombination rates of NW-LEDs between room temperature and $40{ }^{\circ} \mathrm{C}$ was not limited by Shockley-Read-Hall recombination, Auger recombination, or carrier leakage mechanisms, thus realizing droop-free operation. The discovery of reliable, droop-free devices constitutes significant progress toward the development of nanowires for practical applications. Our monolithic approach realized a high-performance device that will revolutionize the way high power, low-junction-temperature LED lamps are manufactured for solid-state lighting and for applications in high-temperature harsh environment.

KEYWORDS: Quantum-disks-in-nanowire, efficiency droop, light-emitting diode, molecular beam epitaxy

$\mathrm{D}$ evices that incorporate III-nitride materials are widely used in solid-state lighting and displays. ${ }^{1}$ However, the application of nitride light-emitting diodes (LEDs) is limited by their decreased efficiency at the peak sensitivity of human photopic vision spectrum, termed the "green gap". ${ }^{2}$ Moreover, the high dislocation density of the materials, together with efficiency droop phenomenon of LEDs at high current injection also hinder their high-brightness applications, which was attributed to the saturation of the radiative recombination rate and increase in the nonradiative recombination rate, such as Shockley-Read-Hall (SRH) nonradiative recombination,
Auger recombination, and electron leakage., ${ }^{3,4}$ Compared to conventional planar devices, III-nitride nanowire devices have attractive advantages, such as dislocation- and strain-free materials, a reduced polarization field, and enhanced carrier confinement. $^{5,6}$ Nanowire devices on silicon have been investigated for application in LEDs and lasers. ${ }^{7,8}$

Received: May 12, 2016

Revised: June 13, 2016

Published: June 28, 2016 

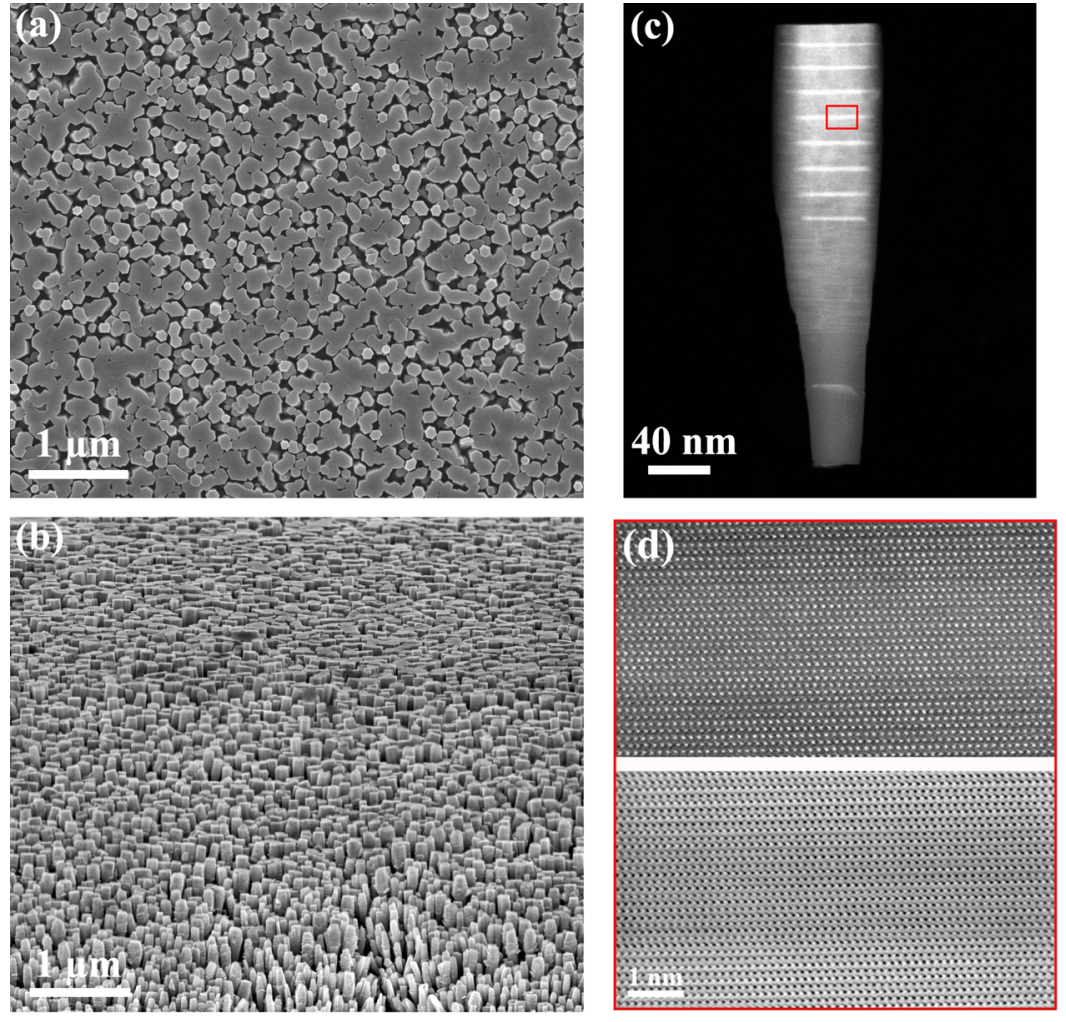

(e)
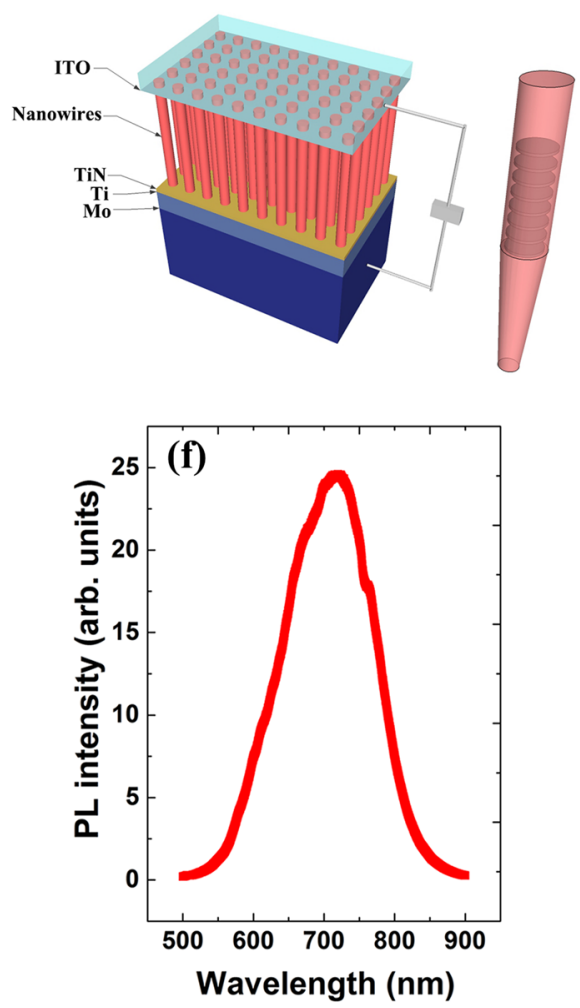

Figure 1. Plan-view (a) and elevation-view (b) SEM images of the Qdisks-in-nanowires; (c) typical HAADF-STEM image of InGaN/GaN Qdisks nanowires, which provides Z-contrast to differentiate the InGaN Qdisks from the GaN barrier; (d) high-magnification HAADF and ABF-STEM images of the Qdisk; (e) a schematic of an LED on Mo substrates; and (f) RT PL spectrum of InGaN/GaN Qdisks in nanowires, excited using 473 nm laser.

While nanowires on silicon have several desirable properties for application in LEDs emitting at true-green $(555 \mathrm{~nm})$ wavelengths and beyond, the formation of an amorphous $\mathrm{SiN}_{x}$ layer at the nanowires-silicon interface impedes heat dissipation and electrical conduction. ${ }^{9}$ The high aspect ratio of nanowires and the laterally discontinuous epitaxy further limits carriers and acoustic phonon conduction, leading to one-dimensional heat transport, ${ }^{10,11}$ severe junction heating, and degradation to performance and lifetimes of nanowire devices. ${ }^{12,13}$ The thermal droop of nanowire LEDs (NW-LEDs) is a considerable drawback for high-power applications at high-current-densities where the operating temperature is above $100{ }^{\circ} \mathrm{C} .{ }^{14,15}$ Moreover, lasers typically operate at higher injection current densities and are even more sensitive to the junction heating. ${ }^{16}$ Therefore, an efficient measurement of nanowire temperature is necessary to produce reliable nanowire-based light-emitters. It is noted that heating at the junctions of nitride devices are typically estimated based on their heat sink temperature, a method that has been studied for planar LEDs ${ }^{17,18}$ but not for NW-LEDs on silicon, which typically have a high turn-on voltage. ${ }^{19}$ In addition, investigation into the reliability and lifetime of NW-LEDs, by means of soft burn-in test, which is important for assessing their practical applications, ${ }^{20}$ has not been studied to the best of our knowledge.

Here, we investigate the temperature-dependent and timeresolved carrier dynamics and the temperature-dependent device measurements of high-power InGaN/GaN quantumdisks (Qdisks)-in-nanowire LEDs on molybdenum (Mo) emitting at $\sim 710 \mathrm{~nm}$, beyond the true-green wavelength. These LEDs exhibited an ultralow turn-on voltage of $\sim 2 \mathrm{~V}$, tested droop-free at an unprecedentedly high-input-powerdensity of $45 \mathrm{~kW} / \mathrm{cm}^{2}(20-\mu \mathrm{m}$ diameter NW-LED), potentially escaping the "valley of droop" for energy-efficient lighting. ${ }^{21}$ The LEDs on an all-metal stack of $\mathrm{TiN} / \mathrm{Ti} / \mathrm{Mo}$ effectively dissipated heat for high-power operations, which resulted in a near-constant junction temperature. This is evident in currentdependent confocal-Raman measurements up to an injection current of $700 \mathrm{~mA}$ on a $380 \mu \mathrm{m} \times 380 \mu \mathrm{m} \mathrm{NW}$-LED, which is attributed to the absence of carrier- and phonon-transport barriers in the monolithic NW-LED/heat-sink platform. The merits of our uncooled operation and reliable droop-free NWLEDs were further accentuated when soft burn-in at a constant current of $600 \mathrm{~mA}$ was applied. The same NW-LED sustained direct-current $(\mathrm{dc})$ electrical-annealing over an $8 \mathrm{~h}$ period, resulting in stable light-output upon burn-in maturity without catastrophic failure. The devices presented herein are hence suitable for the practical and economical deployment of highperformance LEDs and lasers, as well as for cross-disciplinary applications, such as visible-light communications, photovoltaics, photodetection, and sensing in high-temperature harsh environments.

NW-LEDs on Mo substrate were grown using a Veeco Gen 930 plasma-assisted molecular beam epitaxy system as has previously been described. ${ }^{22}$ After the deposition of a $500 \mathrm{~nm}$ thick Ti layer on the Mo substrate using an electron-beam evaporator, eight InGaN-disks $(\sim 3 \mathrm{~nm})$, separated by a GaNbarrier $(\sim 12 \mathrm{~nm})$, followed by a p-GaN layer $(\sim 50 \mathrm{~nm})$, were grown on top of a $150 \mathrm{~nm}$ thick layer of n-type GaN. To compare device performance, NW-LEDs on $\mathrm{Si}$ were also prepared using a $300 \mathrm{~nm}$ thick n-type GaN layer, eight InGaN- 

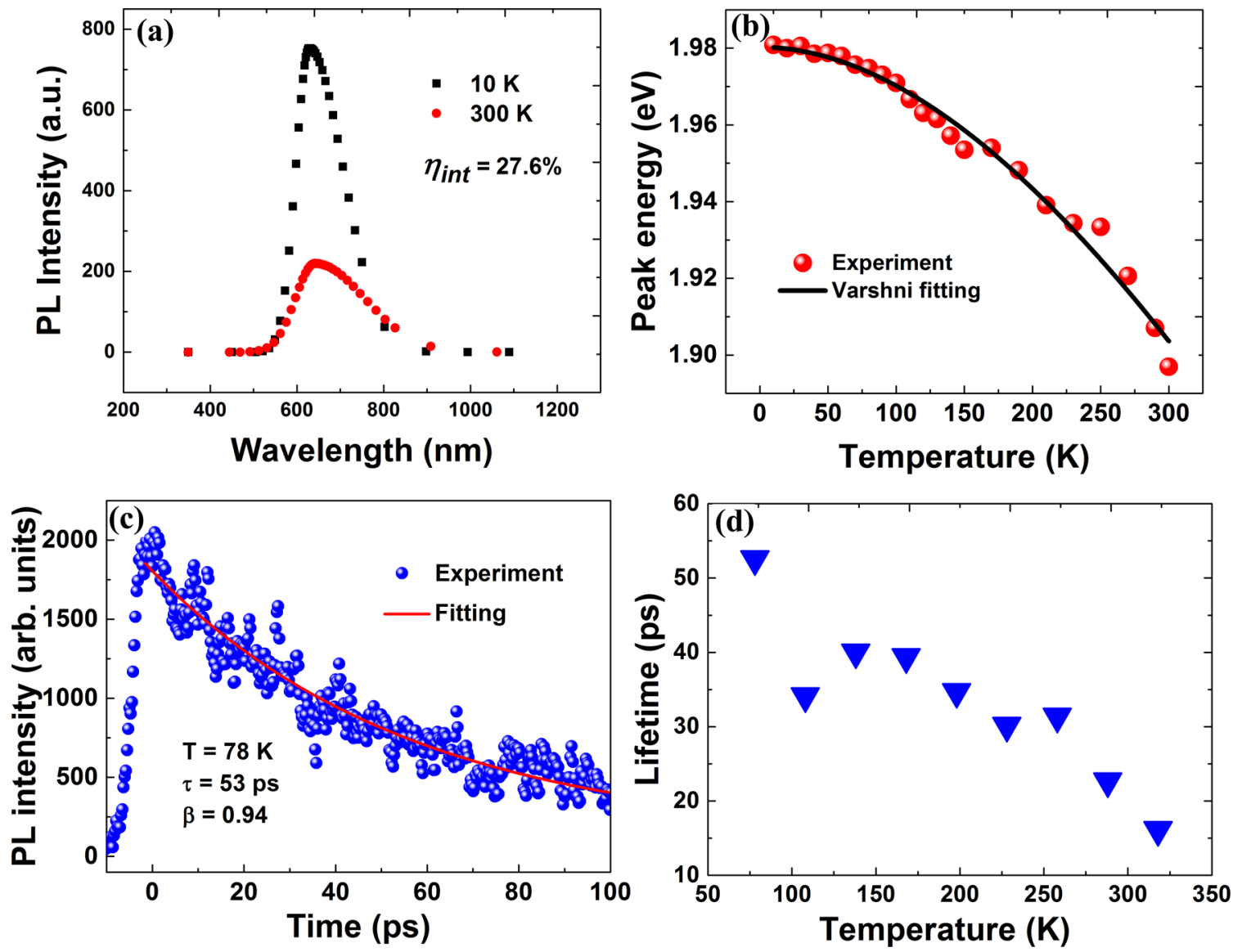

Figure 2. (a) TDPL of Qdisks at 10 and $300 \mathrm{~K}$, excited using $405 \mathrm{~nm}$ laser; (b) the peak energy of Qdisks plotted as a function of temperature, which is fitted using the Varshni equation (solid line). The Varshni parameters used for the fit are $\alpha=0.00112 \mathrm{eV} \mathrm{K}^{-1}$ and $\beta=1011.2 \mathrm{~K}$; (c) typical PL decay for Qdisks; (d) total recombination lifetime derived from TRPL measurements for InGaN Qdisks.

disks $(\sim 3 \mathrm{~nm})$ separated by GaN-barrier $(\sim 12 \mathrm{~nm})$, and a 150 $\mathrm{nm}$ thick p-type GaN layer. After growth, the morphology of nanowires was characterized using an FEI Magellan FEG fieldemission scanning electron microscope (FE-SEM) and a probecorrected FEI Titan operating at $300 \mathrm{kV}$ in high-angle annular dark-field scanning transmission electron microscopy (HAADF-STEM) and annular bright-field (ABF) STEM mode. Microphotoluminescence $(\mu \mathrm{PL})$ was measured using a $473 \mathrm{~nm}$ excitation laser at room temperature (RT) to reveal the emission wavelength of the Qdisks. Temperature-dependent photoluminescence (TDPL) measurements were performed using a closed-loop compressed helium-cooled cryostat with $405 \mathrm{~nm}$ excitation $\mathrm{cw}$ laser from 10 to $300 \mathrm{~K}$. Time-resolved photoluminescence (TRPL) measurements performed with a frequency-doubled $(\lambda=405 \mathrm{~nm})$ mode-locked $\mathrm{Ti}: \mathrm{Al}_{2} \mathrm{O}_{3}$ laser (pulse width $180 \mathrm{fs}$; repetition rate $76 \mathrm{MHz}$ ) from 77 to $300 \mathrm{~K}$ were used to study the carrier lifetimes of Qdisks. TRPL signal was dispersed by a Princeton Instrument SP2300 spectrometer ( 50 grooves $/ \mathrm{mm}$ grating) and then detected by a Hamamatsu C5680 streak camera with $\sim 4$ ps temporal resolution in synchroscan mode. NW-LEDs on Mo of different mesa sizes were fabricated and tested. ${ }^{23}$ The temperature-dependence of devices was tested from -60 to $40{ }^{\circ} \mathrm{C}$ on a Cascade Microtech 11000 probe station to shed light on the origin of droop-free operation. Current-dependent micro-Raman measurements were carried out using below-bandgap laser excitation sources in the backscattering geometry. NW-LEDs on Mo or Si were used to compare the degree of junction heating during operation using the $E_{2}$ high-frequency mode, $E_{2}$ (high), Raman peak shift. Finally, the burn-in tests were also conducted on $380 \mu \mathrm{m}$ square NW-LED to evaluate the reliability of the device.

Figure 1a,b shows the plan-view and elevation-view SEM images of the Qdisks-in-nanowires on Mo. Vertical nanowires were grown epitaxially on the $\mathrm{TiN}$ transition layer (see the Supporting Information for more details on the growth of nanowires, S1). Figure 1a shows that nanowires have hexagonal top surface, an average length of $\sim 300 \mathrm{~nm}$, a lateral size of $50-$ $100 \mathrm{~nm}$ that tapers from the bottoms, and a density of $\sim 1.0 \times$ $10^{10} \mathrm{~cm}^{-2}$. The InGaN/GaN Qdisks embedded in the center of the nanowires with average In composition estimated to be $42.3 \%$ (see the Supporting Information for XRD profiles of the nanowire samples, S1) are clearly visible in Figure 1c, showing a high crystal quality without defects. The diameters increased during growth because of the enhanced lateral growth and increased In incorporation at lower temperatures. To study the distribution of In in the Qdisks, high-resolution HAADF and ABF-STEM were performed. The images in Figure 1d show no obvious contrast variation in the Qdisks, indicating the absence of evident In clustering. Although In-rich clusters within planar quantum wells (QWs) have been reported and contested with the phenomenon attributed to the large miscibility gap of the InGaN alloy, ${ }^{24}$ Kamal et. al, Steffi et. al, and Jung et. al, however, observed the absence of In clustering by TEM and atom probe tomography. ${ }^{25-27}$ It is believed that the high crystal 
quality of nanowires without lattice imperfections prevented the formation of In clusters in the Qdisks. ${ }^{28,29}$

Figure le shows a schematic of the fabricated NW-LEDs. The In composition of the Qdisks was tuned to achieve the peak emission wavelength of $700 \mathrm{~nm}$ (see Figure 1f), which can be reflected by the TiN transition layer underneath the nanowires. This wavelength regime is difficult to achieve in InGaN/GaN planar thin films. However, using the present platform, we achieved a long emission wavelength while simultaneously implementing the n-metal contact, the optical reflector, and the heat sink as soon as the samples were grown, ${ }^{22}$ thus realizing seamless integration of metal and epitaxy, that is, the monolithic metal-optoelectronic.

TDPL was performed to evaluate the internal quantum efficiency of these Qdisks, which was estimated to be $27.6 \%$ using the ratio of integrated PL intensity at $300 \mathrm{~K}$ to that at 10 $\mathrm{K}$ as shown in Figure $2 \mathrm{a}$, assuming that the nonradiative recombination centers are frozen at $10 \mathrm{~K}^{30}$ Figure $2 \mathrm{~b}$ shows the temperature variation of the Qdisks PL peak energy, which was fitted with the Varshni equation. ${ }^{31}$ The absence of the reported S-type behavior indicates there is no obvious carrier localization in the InGaN Qdisks. ${ }^{30}$ Figure $2 \mathrm{c}$ shows the typical Qdisks PL decay curve obtained from TRPL measurements conducted at $405 \mathrm{~nm}$, which was analyzed with the stretched exponential model $I=I_{0} \exp (-t / \tau)^{\beta}$, where $I$ is the measured $\mathrm{PL}$ intensity at time $t, I_{0}$ is the PL peak intensity at $t=0, \tau$ is the total recombination lifetime, and $\beta$ is the stretching parameter. The latter term incorporates the effect of high excitation carrier density and/or disorder within the material, such as compositional variation in nanowires. ${ }^{32}$ The total recombination lifetimes of the Qdisks derived from the analysis are in the range of $16-53$ ps from 77 to $300 \mathrm{~K}$, as shown in Figure $2 \mathrm{~d}$. This short recombination lifetime was attributed to the efficient radiative recombination of photoexcited carriers in the $\mathrm{InGaN}$ Qdisks in the absence of competition from carrier localization states, which is consistent with the TDPL results. ${ }^{33,34}$ The decrease in lifetime with increasing temperature indicates that the nonradiative contribution decreases, which is attributed to surface defects, ${ }^{35}$ and the dramatic increase in lifetime at low temperatures is due to freeze-out of nonradiative recombination centers. $^{36}$ The stretching parameter is between $0.82-0.97$ over a wide range of temperatures due to high excitation carrier density and less compositional disordering within the nanowires. ${ }^{37}$ A more detailed analysis of carrier dynamics, the radiative $\left(\tau_{\mathrm{r}}\right)$ and nonradiative $\left(\tau_{\mathrm{nr}}\right)$ lifetimes, will be published elsewhere in which the significantly smaller $\tau_{\mathrm{r}}$ than those measured in InGaN/GaN QWs or nanowires were attributed to the strain-relaxation-induced reduction in piezoelectric field and improved lateral confinement in dot-like Qdisks, and the short $\tau_{\mathrm{nr}}$ were attributed to surface defects. ${ }^{35-37}$

NW-LEDs on Mo incorporating these nanowires have been fabricated and characterized. Figure 3a shows the light output power-current-voltage $(L-I-V)$ characteristics of an LED with a mesa diameter of $400 \mu \mathrm{m}$ measured under dc injection. The inset shows the charge-coupled device (CCD) image for the uniformly illuminated LED, which has a low turn-on voltage of $\sim 2 \mathrm{~V}$ (see the Supporting Information for a video demonstrating the operation of the NW-LED). The low turnon voltage was achieved due to the absence of interface amorphous layer, which did not form due to the choice of Tibuffer layer, forming a contact layer. In another report, the formed $\mathrm{SiN}_{x}$ amorphous layer can only be removed through the NW-LEDs lift-off process. ${ }^{38}$ In continuous-wave mode, the
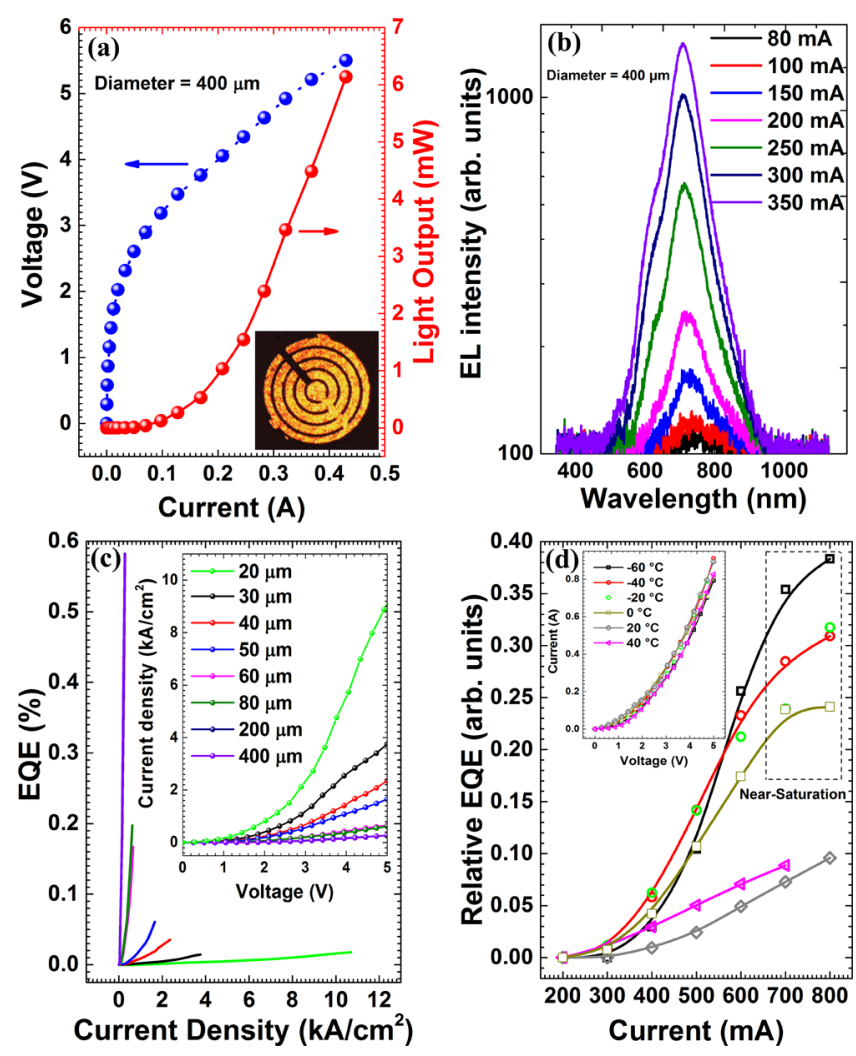

Figure 3. (a) The measured $L-I-V$ characteristics of the $400 \mu \mathrm{m}$ diameter LED; the inset shows the optical microscope image of the illuminated LED; (b) the EL spectra of the LED at injection currents from $80-350 \mathrm{~mA}$; (c) the EQE of LEDs with different sizes; the inset shows the measured $I-V$ characteristics of LEDs; (d) EQE with current injection between -60 and $40{ }^{\circ} \mathrm{C}$; the inset shows the $I-V$ characteristics of LEDs between -60 and $40^{\circ} \mathrm{C}$. The dashed rectangle indicates the region where the relative $\mathrm{EQE}$ reached near-saturation.

light output power was up to $6.14 \mathrm{~mW}$ when operating at 500 $\mathrm{mA}$ injection current and $\sim 5 \mathrm{~V}$ forward bias voltage. Utilizing the beneficial effect of lateral strain relief in nanowires, we achieved peak emission wavelength at $\sim 710 \mathrm{~nm}$ with negligible blueshift in the injection current range of $80-350 \mathrm{~mA}$ due to the reduced polarization field (see Figure $3 b$ showing the RT electroluminescence (EL) spectra of the NW-LEDs with increasing injection current densities). The output power of planar far-red $(740 \mathrm{~nm})$ LED grown on sapphire has been reported to be $2 \mu \mathrm{W} .^{39}$ Later, Hwang et al. reported an LED with an output power of $1.1 \mathrm{~mW}$ emitting at $629 \mathrm{~nm} .{ }^{40}$ For NW-LEDs, the piezoelectric field decreases, as compared to planar LEDs, due to lateral strain relaxation. However, their output power is reported to remain within nanowatts or microwatts for the green color. ${ }^{41} \mathrm{Mi}$ et al. demonstrated highpower InGaN/GaN/AlGaN core-shell NW-LEDs with output powers up to $\sim 1.5 \mathrm{~mW}$ for emission at $\sim 630 \mathrm{~nm}$ measured in pulse mode. ${ }^{42}$ The optical power achieved in this investigation is considerably higher than previous reports on long-wavelength NW-LEDs or planar LEDs despite the high In composition that reduces the overlap of electron-hole wave functions (see the Supporting Information for the Nextnano simulation of the band diagram of red LEDs, S2). ${ }^{43}$

The inset in Figure $3 c$ shows the measured injection current density versus voltage characteristics of LEDs different sizes. The LEDs with smaller sizes showed lower forward voltages and lower series resistances. When operating at $5 \mathrm{~V}$, the 


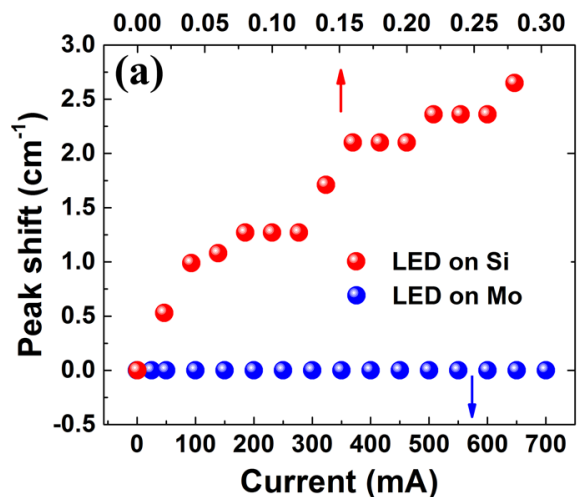

(b)

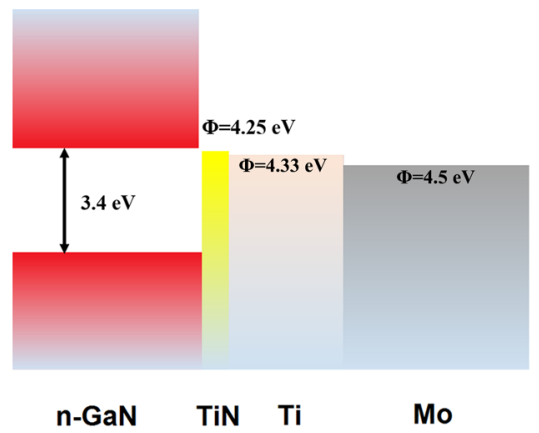

(c)

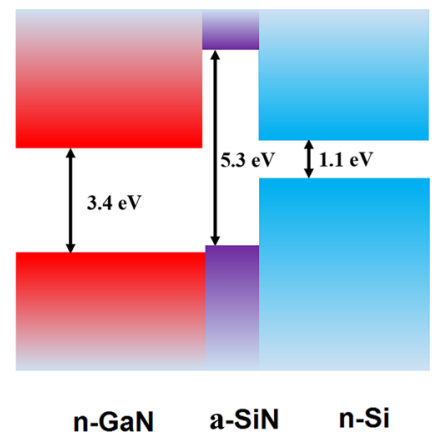

Figure 4. (a) Current-dependent Raman measurements of LEDs on Mo and Si; (b,c) energy band alignment of n-GaN on Mo (b) and Si (c).

injection current density of the $20 \mu \mathrm{m}$ NW-LED reaches $9 \mathrm{kA} /$ $\mathrm{cm}^{2}$, while that of the $400 \mu \mathrm{m} \mathrm{NW-LED} \mathrm{is} \mathrm{only} 280 \mathrm{~A} / \mathrm{cm}^{2}$ due to the effect of disparate current spreading. ${ }^{44,45}$ Figure $3 \mathrm{c}$ also shows the external quantum efficiency (EQE) of LEDs with different sizes. The LEDs with smaller sizes have a lower EQE, probably because they dissipate heat less efficiently. ${ }^{46}$ Moreover, there is no efficiency droop for LEDs of any size. Several reports have shown that that EQE tends to increase with increasing current injection for NW-LEDs, which has been attributed to near-dislocation- and strain-free nanowires. ${ }^{47,48}$ The argument of dislocation- and strain-free nanowires seems to correlate well with the above results, which we obtained for the Qdisks-in-nanowires grown on Mo. In fact, the efficiencydroop free phenomenon was even attainable at the unprecedented high-input power density of $45 \mathrm{~kW} / \mathrm{cm}^{2}$. By operating beyond the "valley of droop", these NW-LEDs are practical and economical options for application in solid-state lighting. ${ }^{21}$ Moreover, because differently sized LEDs were fabricated from the same nanowire epitaxy, we can exclude the possibility of surface-states related SRH process, because the EQE slopes are different at low current density where SRH dominates (see Figure 3c).

To investigate this droop-free mechanism, we conducted a low-temperature device test. As shown in the inset in Figure 3d, the NW-LED showed similar $I-V$ characteristics between -60 and $40{ }^{\circ} \mathrm{C}$ at a turn-on voltage of $2 \mathrm{~V}$. The EQE increased as the temperature decreased due to reduced SRH nonradiative recombination (see Figure $3 \mathrm{~d}$ and the Supporting Information for EL spectra of the LED at an injection current of $600 \mathrm{~mA}$ and various temperatures, S3). Moreover, below RT the EQE showed near-saturation. It is known that the radiative recombination rate is enhanced and $\mathrm{SRH}$ nonradiative recombination is suppressed at low temperature. ${ }^{49,50}$ Because of the absence of In clusters, dislocations, and the reduced polarization field, it is difficult to attribute them to the efficiency droop. The monotonic increase of EQE at RT and above and the presence of the peak EQE at lower temperatures suggest that the mechanism became stronger at lower temperatures, different from reports on Auger recombination. ${ }^{51}$ Moreover, the high-quality nanowires grown on our high-thermalconductivity platform also likely mitigated the effect of phonon-assisted Auger recombination. ${ }^{52,53}$ Thus, we can also exclude Auger recombination as the dominant reason. At low temperatures, it is easier for the holes than the electrons to freeze-out because $\mathrm{Mg}$ acceptors have higher ionization energy. As a result, the Qdisks are utilized less, causing the effective active volume reduction and saturation of the radiative recombination rate. Because of the higher concentration of electrons and the associated high mobility as compare to that of holes, the electrons do not recombine in the Qdisks overflow to the p-GaN layer. ${ }^{54}$ The competition between carrier leakage and change in radiative recombination rate are believed to cause near-saturation of device efficiency below RT. ${ }^{55}$ The results from the TEM, TDPL, and TRPL evidence that carrier localization caused by In clustering is absent, leading to a uniform carrier distribution in high-quality Qdisks, hence increasing the effective active volume in InGaN Qdisks. ${ }^{56}$ Ultimately, the saturation of the radiative recombination rate is suppressed, ${ }^{4}$ and at RT or above this is not limited by SRH nonradiative recombination, Auger recombination, or carrier leakage, thus realizing droop-free operation.

To further study the phonon-assisted Auger recombination in our NW-LEDs on Mo, we measured the junction heating of the LEDs while under DC operation using micro-Raman spectroscopy. This is based on the relative downshift of the $\mathrm{GaN} \mathrm{E}_{2}$ (high) phonon Raman peak of the LEDs under current injection with respect to its peak position at zero current (see Supporting Information for the setup of the current dependent Raman measurements, S4). ${ }^{17,57}$ A calibration measurement was also performed to illustrate the relative shift of the $\mathrm{E}_{2}$ (high) phonon peak of a $\mathrm{GaN}$ layer at a given temperature with respect to the peak position at RT (see Supporting Information for the temperature-dependent Raman measurements, S5). ${ }^{18}$ For comparison, LEDs on $\mathrm{Si}$ were also characterized in the same way. By using below-bandgap laser excitations, we prevented the absorption of laser light and thermal heating. Because heat is stored in the optical phonon bath, it is valid for us to estimate the heat generated by Raman spectroscopy. The generated optical phonons decayed into acoustic phonons through anharmonic processes, which are then removed from the hot spot because of their large group velocity. ${ }^{58,59}$ As shown in Figure 4a, LEDs on Mo showed almost constant temperature while operating across the full injection range. Because of the existence of the SiN amorphous layer of LEDs on $\mathrm{Si}$, there is a barrier for the carriers which is absent for LEDs on Mo, as shown in the energy band alignment in Figure 4b,c. Moreover, because of the highly mismatched specific heat, group velocity, and density between $\mathrm{GaN}$ and amorphous $\mathrm{SiN}$, phonon ballistic transport across the interface is greatly affected. ${ }^{60,61}$ Further investigation is necessary to determine the role of the applied electric field in carrier and phonon transport.

The operating lifetime of NW-LEDs is an important parameter for practical applications but has not been studied before. To evaluate the reliability of our devices to operate at 

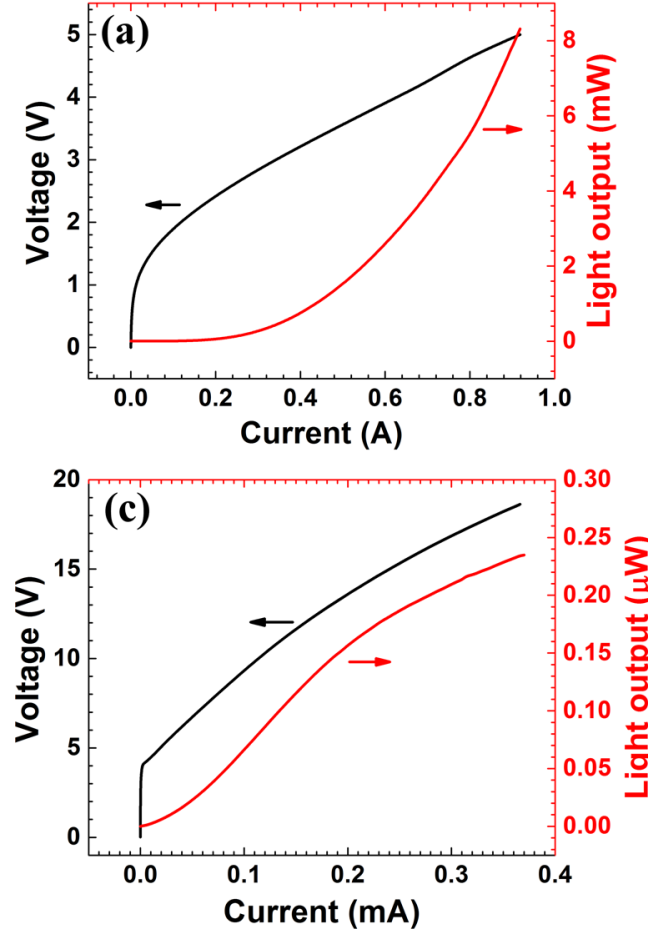
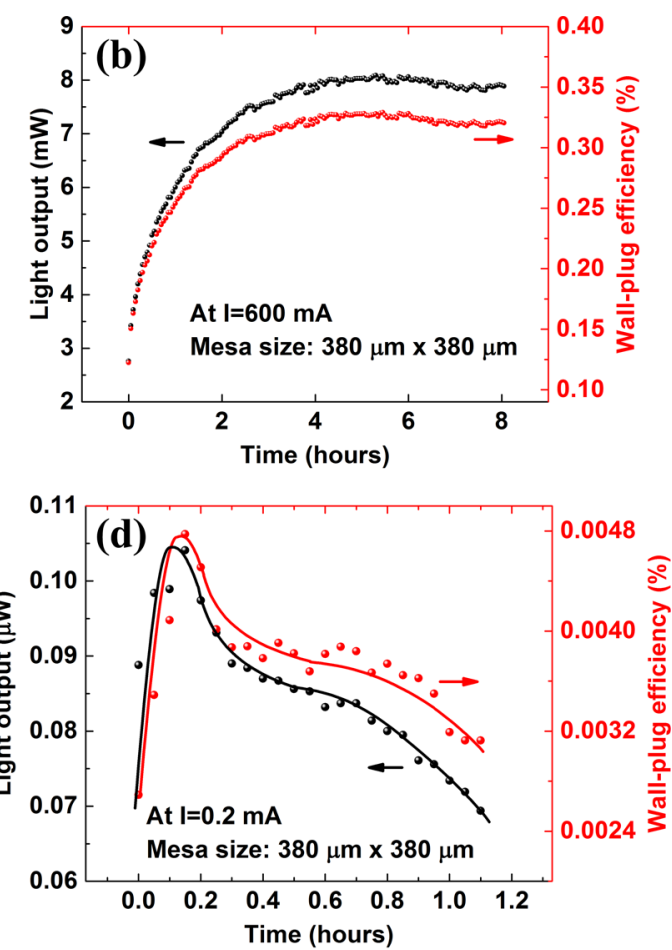

Figure 5. (a) Typical $L-I-V$ of LEDs on Mo; (b) light output power and wall-plug efficiency of LEDs on Mo versus time. A constant current of 600 $\mathrm{mA}$ was applied to the LEDs with size of $380 \mu \mathrm{m} \times 380 \mu \mathrm{m}$; (c) typical $L-I-V$ of LEDs on Si; (d) light output power and wall-plug efficiency of LEDs on Si versus time. A constant current of $0.2 \mathrm{~mA}$ was applied to the LEDs with size of $380 \mu \mathrm{m} \times 380 \mu \mathrm{m}$.

high current injections, light output power and wall-plug efficiency were plotted versus time. Figure 5a,c shows the typical $L-I-V$ characteristics of LEDs on Mo or Si. Constant currents of 600 and $0.2 \mathrm{~mA}$ were applied to the LEDs on Mo and $\mathrm{Si}$ with sizes of $380 \mu \mathrm{m} \times 380 \mu \mathrm{m}$, respectively, and the voltage and output power were recorded intermittently. The output power and wall-plug efficiencies of LED on Mo were stronger than those on $\mathrm{Si}$, as shown in Figure 5b,d. We noted that the output power and efficiency of LEDs on Mo steadily increased with time and after $3.3 \mathrm{~h}$ reached a stable level of power and efficiency. The results show that the LED presented in this work is capable of running continuously for a minimum of $8 \mathrm{~h}$, setting a precedence in NW-LED longevity. Because of the excellent thermal conductivity of LEDs on Mo, the heat generated, even at high current injection, could be dissipated efficiently without degrading device performance. Such electrical annealing improves the crystal quality of nitrides dramatically and thus improves device performance with time. ${ }^{13}$ In contrast, the output power and efficiency of LEDs on $\mathrm{Si}$ increased at the beginning due to electrical annealing and then deteriorated gradually after $0.17 \mathrm{~h}$, burning out after $1.1 \mathrm{~h}$. Because of their poor thermal conductivity, the heat generated could not be dissipated efficiently, causing overheating and damage to the device.

In conclusion, we demonstrated droop-free, low turn-on voltage, uncooled, and reliable operation of high-power NWLEDs emitting at $\sim 710 \mathrm{~nm}$. The junction temperature of NWLEDs on Mo while operating was almost constant up to a 700$\mathrm{mA}$ injection current, owing to the absence of a carrier and phonon transport barrier on the all-metal stack of TiN/Ti/Mo. We further performed soft burn-in at a constant current of 600 $\mathrm{mA}$ for the InGaN/GaN Qdisks-in-nanowire-LEDs with a size of $380 \mu \mathrm{m} \times 380 \mu \mathrm{m}$ and achieved a minimum of $8 \mathrm{~h}$ operation. The high current operation was enabled using monolithic building block comprising low turn-on voltage NWLEDs epitaxially grown on Mo. Our investigations highlighted the considerable strength of the metal-optoelectronic solution to achieve practical high-power NW-LEDs for applications at the true-green wavelength and beyond. Moving forward, there is potential for the development of long-wavelength NW-LEDs and lasers and a plethora of high-performance multidisciplinary applications in the field of visible-light communications and photovoltaics, as well as photodetection and sensing in hightemperature harsh environments.

\section{ASSOCIATED CONTENT}

\section{S Supporting Information}

The Supporting Information is available free of charge on the ACS Publications website at DOI: 10.1021/acs.nanolett.6b01945.

Details on the growth of InGaN/GaN quantum-disks-innanowires on $\mathrm{Ti} / \mathrm{Mo}$, Nextnano simulation of band diagram of red LEDs, EL spectra of the LED at an injection current of $600 \mathrm{~mA}$ at different temperatures, and the setup for the current-dependent Raman measurements, temperature-dependent Raman measurements of LEDs on sapphire, Si, and Mo.(PDF)

The video demonstrates the operation of NW-LEDs. (AVI)

\section{AUTHOR INFORMATION}

\section{Corresponding Authors}

*E-mail: tienkhee.ng@kaust.edu.sa.

*E-mail: boon.ooi@kaust.edu.sa.

\section{Author Contributions}

C.Z. and T.K.N. contributed equally. C.Z. and T.K.N. conceived of the idea, designed the investigations, the growth 
experiments, and the fabrication process. C.Z. performed the molecular beam epitaxial growth. C.Z., A.P., G.B.C., C.S., and J.E. fabricated and tested the devices. C.Z. and G.B.C. performed Raman characterizations. R.T.E., I.A.A., and I.S.R. performed the TRPL measurements and analyses. B.J. performed the Nextnano simulation. C.Z., T.K.N., and B.S.O. wrote the manuscript. T.K.N. led the molecular bean epitaxy program. B.S.O., A.Y.A., and M.M.E. supervised the team. All authors have read, contributed to, and approved the final version of the manuscript.

\section{Notes}

The authors declare no competing financial interest.

\section{ACKNOWLEDGMENTS}

The authors acknowledge funding support from King Abdulaziz City for Science and Technology (KACST) Technology Innovation Center (TIC) for Solid State Lighting (KACST TIC R2-FP-008), and KAUST baseline funding (BAS/1/161401-01).

\section{REFERENCES}

(1) Nakamura, S.; Fasol, G. The Blue Laser Diode: GaN Based Light Emitters and Lasers; Springer: New York, 1997.

(2) Yang, Y.; Cao, X. A.; Yan, C. H. Appl. Phys. Lett. 2009, 94, 041117.

(3) Piprek, J. Phys. Status Solidi A 2010, 207, 2217-2225.

(4) Shim, J.-I.; Kim, H.; Han, D.-P.; Shin, D.-S.; Kim, K. S. Proc. SPIE 2014, 8986, 89861S-89861S-8.

(5) Wang, P.; Yuan, Y.; Zhao, C.; Wang, X.; Zheng, X.; Rong, X.; Wang, T.; Sheng, B.; Wang, Q.; Zhang, Y.; Bian, L.; Yang, X.; Xu, F.; Qin, Z.; Li, X.; Zhang, X.; Shen, B. Nano Lett. 2016, 16, 1328-1334.

(6) Zhao, C.; Chen, Y. H.; Xu, B.; Jin, P.; Wang, Z. G. Appl. Phys. Lett. 2007, 91, 033112.

(7) Frost, T.; Jahangir, S.; Stark, E.; Deshpande, S.; Hazari, A.; Zhao, C.; Ooi, B. S.; Bhattacharya, P. Nano Lett. 2014, 14, 4535-4541.

(8) Ng, T. K.; Zhao, C.; Shen, C.; Jahangir, S.; Janjua, B.; Slimane, A. B.; Kang, C. H.; Syed, A. A.; Li, J.; Alyamani, A. Y. CLEO: Science and Innovations 2014, SM2J.2.

(9) Hestroffer, K.; Leclere, C.; Cantelli, V.; Bougerol, C.; Renevier, H.; Daudin, B. Appl. Phys. Lett. 2012, 100, 212107.

(10) Matthews, J.; Hoffmann, E. A.; Weber, C.; Wacker, A.; Linke, H. Phys. Rev. B: Condens. Matter Mater. Phys. 2012, 86, 174302.

(11) Jen-Hau, C.; Seghete, D.; George, S. M.; Yang, R.; Lee, Y. C. In Micro Electro Mechanical Systems (MEMS); 2010 IEEE 23rd International Conference, Wanchai, Hong Kong; IEEE: Piscataway, NJ, 2010; pp 468-471.

(12) Li, Q.; Westlake, K. R.; Crawford, M. H.; Lee, S. R.; Koleske, D. D.; Figiel, J. J.; Cross, K. C.; Fathololoumi, S.; Mi, Z.; Wang, G. T. Opt. Express 2011, 19, 25528-25534.

(13) Tan, B. S.; Yuan, S.; Kang, X. J. Appl. Phys. Lett. 2004, 84, 27572759.

(14) Arik, M.; Becker, C. A.; Weaver, S. E.; Petroski, J. Proc. SPIE 2003, 5187, 64-75.

(15) Pan, C.-C.; Gilbert, T.; Pfaff, N.; Tanaka, S.; Zhao, Y.; Feezell, D.; Speck, J. S.; Nakamura, S.; DenBaars, S. P. Appl. Phys. Express 2012, 5, 102103.

(16) Cao, X. A.; Arthur, S. D. Appl. Phys. Lett. 2004, 85, 3971-3973. (17) Alarcón-Lladó, E.; Bin-Dolmanan, S.; Lin, V. K. X.; Teo, S. L.; Dadgar, A.; Krost, A.; Tripathy, S. J. Appl. Phys. 2010, 108, 114501.

(18) Chitnis, A.; Sun, J.; Mandavilli, V.; Pachipulusu, R.; Wu, S.; Gaevski, M.; Adivarahan, V.; Zhang, J. P.; Khan, M. A.; Sarua, A.; Kuball, M. Appl. Phys. Lett. 2002, 81, 3491-3493.

(19) Shaofei, Z.; Connie, A. T.; Laleyan, D. A.; Hieu Pham Trung, N.; Qi, W.; Jun, S.; Ishiang, S.; Zetian, M. IEEE J. Quantum Electron. 2014, 50, 483-490.
(20) Liu, J. S.; Shan, C. X.; Shen, H.; Li, B. H.; Zhang, Z. Z.; Liu, L.; Zhang, L. G.; Shen, D. Z. Appl. Phys. Lett. 2012, 101, 011106.

(21) Wierer, J. J.; Tsao, J. Y.; Sizov, D. S. Laser Photon. Rev. 2013, 7, 963-993.

(22) Zhao, C.; Ng, T. K.; Wei, N.; Prabaswara, A.; Alias, M. S.; Janjua, B.; Shen, C.; Ooi, B. S. Nano Lett. 2016, 16, 1056-1063.

(23) Zhao, C.; Ng, T. K.; Prabaswara, A.; Conroy, M.; Jahangir, S.; Frost, T.; O’Connell, J.; Holmes, J. D.; Parbrook, P. J.; Bhattacharya, P.; Ooi, B. S. Nanoscale 2015, 7, 16658-16665.

(24) Narukawa, Y.; Kawakami, Y.; Funato, M.; Fujita, S.; Fujita, S.; Nakamura, S. Appl. Phys. Lett. 1997, 70, 981-983.

(25) Baloch, K. H.; Johnston-Peck, A. C.; Kisslinger, K.; Stach, E. A.; Gradečak, S. Appl. Phys. Lett. 2013, 102, 191910.

(26) Woo, S. Y.; Bugnet, M.; Nguyen, H. P. T.; Mi, Z.; Botton, G. A. Nano Lett. 2015, 15, 6413-6418.

(27) Jung, W.-Y.; Seol, J.-B.; Kwak, C.-M.; Park, C.-G. Appl. Phys. Lett. 2016, 108, 113111.

(28) Stringfellow, G. B. J. Cryst. Growth 2010, 312, 735-749.

(29) Lei, H.; Chen, J.; Ruterana, P. J. Appl. Phys. 2010, 108, 103503.

(30) Deshpande, S.; Heo, J.; Das, A.; Bhattacharya, P. Nat. Commun. 2013, 4, 1675.

(31) Varshni, Y. P. Physica 1967, 34, 149-154.

(32) Krestnikov, I. L.; Ledentsov, N. N.; Hoffmann, A.; Bimberg, D.; Sakharov, A. V.; Lundin, W. V.; Tsatsul'nikov, A. F.; Usikov, A. S.; Alferov, Z. I.; Musikhin, Y. G.; Gerthsen, D. Phys. Rev. B: Condens. Matter Mater. Phys. 2002, 66, 155310.

(33) Ebaid, M.; Kang, J.-H.; Lim, S.-H.; Ko, S.-M.; Cho, Y.-H.; Ryu, S.-W. Acta Mater. 2014, 65, 118-124.

(34) Jeong, H.; Jeong, H. J.; Oh, H. M.; Hong, C. H.; Suh, E. K.; Lerondel, G.; Jeong, M. S. Sci. Rep. 2015, 5, 9373.

(35) Guo, W.; Zhang, M.; Banerjee, A.; Bhattacharya, P. Nano Lett. 2010, 10, 3355-3359.

(36) Jahangir, S.; Mandl, M.; Strassburg, M.; Bhattacharya, P. Appl. Phys. Lett. 2013, 102, 071101.

(37) Chichibu, S.; Onuma, T.; Sota, T.; DenBaars, S. P.; Nakamura, S.; Kitamura, T.; Ishida, Y.; Okumura, H. J. Appl. Phys. 2003, 93, 2051-2054.

(38) Park, Y.; Jahangir, S.; Park, Y.; Bhattacharya, P.; Heo, J. Opt. Express 2015, 23, A650-A656.

(39) Ohkawa, K.; Watanabe, T.; Sakamoto, M.; Hirako, A.; Deura, M. J. Cryst. Growth 2012, 343, 13-16.

(40) Hwang, J.-I.; Hashimoto, R.; Saito, S.; Nunoue, S. Appl. Phys. Express 2014, 7, 071003.

(41) Bavencove, A. L.; Tourbot, G.; Garcia, J.; Désières, Y.; Gilet, P.; Levy, F.; André, B.; Gayral, B.; Daudin, B.; Le Si, D. Nanotechnology 2011, 22, 345705.

(42) Mi, Z.; Nguyen, H. P. T.; Djavid, M.; Zhang, S.; Connie, A. T.; Sadaf, S. M.; Wang, Q.; Zhao, S.; Shih, I. ECS Trans. 2014, 61, 9-15.

(43) Marquardt, O.; Geelhaar, L.; Brandt, O. J. Comput. Electron. 2015, 14, 464-468.

(44) Horng, R.-H.; Chen, K.Y.; Tien, C.-H.; Liao, J.-C. J. Disp. Technol. 2015, 11, 1010-1013.

(45) Kim, H.; Cho, J.; Lee, J. W.; Yoon, S.; Kim, H.; Sone, C.; Park, Y.; Seong, T.-Y. Appl. Phys. Lett. 2007, 90, 063510.

(46) Lee, H. K.; Yu, J. S. Jpn. J. Appl. Phys. 2010, 49, 04DG11.

(47) Guo, W.; Banerjee, A.; Bhattacharya, P.; Ooi, B. S. Appl. Phys. Lett. 2011, 98, 193102.

(48) Nguyen, H. P. T.; Zhang, S.; Cui, K.; Han, X.; Fathololoumi, S.; Couillard, M.; Botton, G. A.; Mi, Z. Nano Lett. 2011, 11, 1919-1924.

(49) Dmitriev, A.; Oruzheinikov, A. J. Appl. Phys. 1999, 86, 32413246.

(50) Deshpande, S.; Frost, T.; Yan, L.; Jahangir, S.; Hazari, A.; Liu, X.; Mirecki-Millunchick, J.; Mi, Z.; Bhattacharya, P. Nano Lett. 2015, $15,1647-1653$

(51) Galler, B.; Drechsel, P.; Monnard, R.; Rode, P.; Stauss, P.; Froehlich, S.; Bergbauer, W.; Binder, M.; Sabathil, M.; Hahn, B.; Wagner, J. Appl. Phys. Lett. 2012, 101, 131111.

(52) Kioupakis, E.; Rinke, P.; Delaney, K. T.; Van de Walle, C. G. Appl. Phys. Lett. 2011, 98, 161107. 
(53) Pfaff, N. A. Thermal Performance of III-Nitride Light Emitting Diodes and Developments in Laser Based White Lighting. Ph.D. Thesis, University of California, Santa Barbara, 2013.

(54) Shin, D.-S.; Han, D.-P.; Oh, J.-Y.; Shim, J.-I. Appl. Phys. Lett. 2012, 100, 153506

(55) Shim, J.-I.; Han, D.-P.; Kim, H.; Shin, D.-S.; Lin, G.-B.; Meyaard, D. S.; Shan, Q.; Cho, J.; Fred Schubert, E.; Shim, H.; Sone, C. Appl. Phys. Lett. 2012, 100, 111106.

(56) Ryu, H.-Y.; Shin, D.-S.; Shim, J.-I. Appl. Phys. Lett. 2012, 100, 131109.

(57) Schwegler, V.; Seyboth, M.; Schad, S.; Scherer, M.; Kirchner, C.; Kamp, M.; Stempfle, U.; Limmer, W.; Sauer, R. MRS Online Proc. Libr. 1999, 595, F99W11.18.

(58) Vasileska, D.; Hossain, A.; Raleva, K.; Goodnick, S. M. J. Comput. Electron. 2010, 9, 180-186.

(59) Xiong, D.; Zhang, Y.; Zhao, H. Phys. Rev. E 2013, 88, 052128. (60) Chen, G. Phys. Rev. B: Condens. Matter Mater. Phys. 1998, 57, 14958-14973.

(61) Zink, B. L.; Hellman, F. Solid State Commun. 2004, 129, 199204. 